\title{
Properties of Polydisperse Tin-doped Dysprosium and Indium Oxides
}

\author{
Tatyana Malinovskaya ${ }^{1}$, Valentin Suslyaev ${ }^{2}$, Valentina Zhek ${ }^{1}$, Ilya Lysak $^{1,3,{ }^{*}}$, Sergey Melentyev ${ }^{4}$, Kirill Dorozhkin ${ }^{2}$, \\ Eugeny Korovin ${ }^{2}$, and Sergey Pavlov ${ }^{4}$ \\ ${ }^{1}$ Siberian Physical Technical Institute at Tomsk State University, Tomsk, Russian Federation \\ ${ }^{2}$ National Research Tomsk State University, Tomsk, Russian Federation \\ ${ }^{3}$ National Research Tomsk Polytechnic University, Tomsk, Russian Federation \\ ${ }^{4}$ Tomsk State University of Architecture and Building, Tomsk, Russian Federation
}

\begin{abstract}
The results of investigations of the complex permittivity, diffuse-reflectance, and characteristics of crystal lattices of tin-doped indium and dysprosium oxides are presented. Using the methods of spectroscopy and X-ray diffraction analysis, it is shown that doping of indium oxide with tin results in a significant increase of the components of the indium oxide complex permittivity and an appearance of the plasma resonance in its diffuse-reflectance spectra. This indicates the appearance of charge carriers with the concentration of more than $10^{21} \mathrm{~cm}^{-3}$ in the materials. On the other hand, doping of the dysprosium oxide with the same amount of tin has no effect on its optical and electromagnetic properties.
\end{abstract}

\section{Introduction}

Development of modern electronics leads to the need of searching for new promising materials, which are characterised by high chemical and thermal resistance, high permittivity $(\varepsilon=8-20)$ and resistivity $\left(\rho=10^{13}\right.$ $\left.10^{16} \Omega \cdot \mathrm{cm}\right)$. Among them are rare-earth elements oxides (REO). Doping of oxides with various chemical elements allows modifying these parameters over a wide range. As an important material for electronics and optics, dysprosium oxide is also used for the manufacture of optical and magnetic memory and ultrapowerful magnets, as a neutron-absorbing material in nuclear power industry, as a component of redemitting phosphors, and as a component of catalytic systems [1]. Therefore, studies of the dysprosium oxide doping with various elements are of considerable practical interest. The use of such dopants as $\mathrm{Ce}, \mathrm{Rh}, \mathrm{Zr}$, and $\mathrm{Hf}[2-5]$ is well known, but there are practically no data on doping of dysprosium oxide with Sn. At the same time, the most widely studied oxide material used in electronics is indium oxide doped with tin (ITO), which is a solid solution of indium oxide (III) and tin (IV). An optimal weight ratio is $90 \% \mathrm{In}_{2} \mathrm{O}_{3}$ and $10 \%$ $\mathrm{SnO}_{2}$ by [6]. Doping with tin allows increasing concentration of charge carriers in indium oxide up to $10^{21} \mathrm{~cm}^{-3}$ due to the shallow impurity levels formed in its energy-band structure, which significantly affects the electrical and other properties of $\operatorname{In}_{2} \mathrm{O}_{3}$.

Thus, the aim of this work was to study the effect of tin-doping on the properties of indium and dysprosium oxides.

\section{Experimental part}

For the analysis of samples of oxides, the X-ray diffraction method was used. Investigations were carried out on a diffractometer Rigaku Miniflex 600 (USA) using $\mathrm{CuK} \alpha$-radiation in the range of $10^{\circ}-90^{\circ}(2 \theta)$ with the scanning step of $0.02^{\circ}$ and rate of $2 \% \mathrm{~min}$. The identification of the diffraction peaks was performed using JCPDS database. The spectral dependences of the samples diffuse reflectance were recorded using a spectrophotometer IKS-31 (Russia) with photometric sphere and hemisphere. The spectra of complex permittivity (CP) in the frequency range of $115-720 \mathrm{GHz}$ were measured using the "free space" method with a terahertz spectrometer STD-21 (Russia) [7]. Three backward-wave tubes were used to cover the entire range: $115-258,220-511$, and 430-720 GHz. Measurements were conducted on the $0.26 \mathrm{~mm}$ thick planar samples obtained by introducing the oxide powders in a bonding matrix. As the binder, a twocomponent polyurethane varnish brand Kontracid D3010 was used, the rationale for the choice of which and the coating manufacturing technique based on it are described in [8].

\section{Results and discussion}

It was found that the most complete coprecipitation of dysprosium and tin from theirs nitrate solutions takes place at $\mathrm{pH}$ values of 7-10, and that of indium and tin at $\mathrm{pH}$ values of 6-10 (Figure 1). Thus, for doping of dysprosium and indium oxides with tin, we used the method of chemical coprecipitation of dysprosium or indium hydroxides coupled with tin hydroxide from the

Corresponding author: lysak ia@mail.com

(C) The Authors, published by EDP Sciences. This is an open access article distributed under the terms of the Creative Commons Attribution License 4.0 (http://creativecommons.org/licenses/by/4.0/). 
nitrate solutions at $\mathrm{pH} 7$ with subsequent heat treatment in the air at $1000{ }^{\circ} \mathrm{C}$ for 1 hour. The tin content was 10

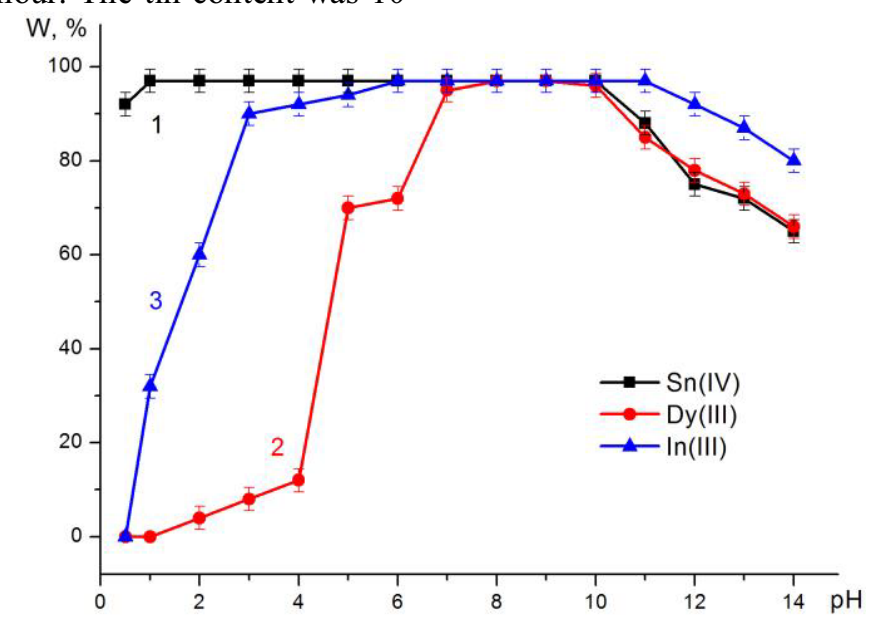

Fig. 1. Dependence of the deposition degree $(W, \%)$ with an aqueous solution of ammonia on the $\mathrm{pH}$ value of the nitrate solution: 1 - Sn(IV); 2 - Dy(III); 3 - In(III)

Diffractograms of ITO powders synthesized as described above were studied in [9]. The studies indicated that after the heat treatment of the coprecipitated indium and tin hydroxides for 1 hour in the air at $1000{ }^{\circ} \mathrm{C}$, the formation of an $\operatorname{In}_{2} \mathrm{O}_{3}$ structure of cubic modification with the increased, compared to the standard values, interplanar distances was observed. Diffraction patterns of powders obtained as a result of heat treatment of dysprosium hydroxide and of coprecipitated dysprosium and tin hydroxides are presented in Figures 2 and 3, respectively. Comparison of the diffraction patterns with the standard data from the database JSPDS presented in these figures in the form of bar graphs suggests that the investigated powders are the dysprosium oxides of cubic modification with the lattice constants $1.0655767 \mathrm{~nm}$ and $1.0640638 \mathrm{~nm}$ for the wt.\%. As the precipitant, $25 \%$ ammonia water was used.

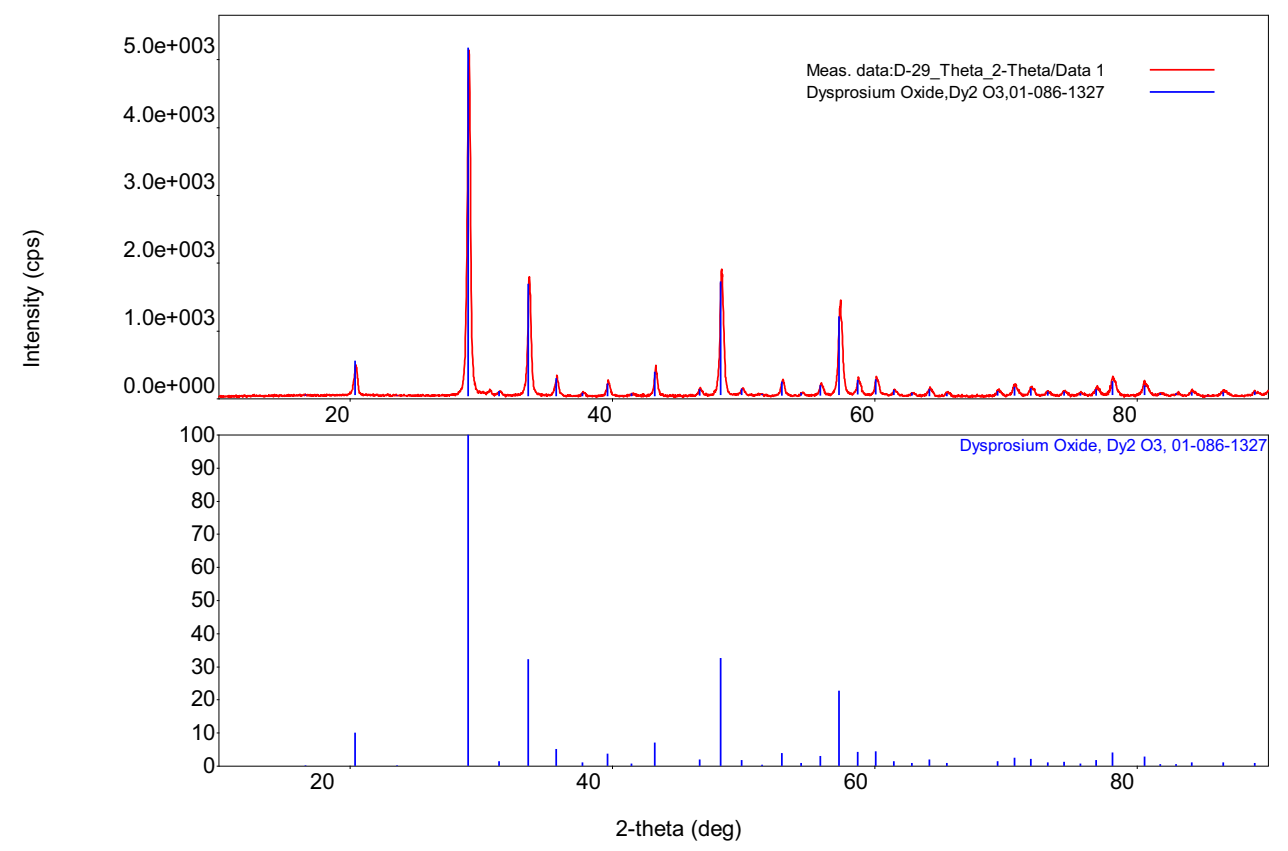

Fig. 2. X-Ray diffraction pattern of the powder obtained by heat treatment at $1000{ }^{\circ} \mathrm{C}$ for 1 hour of dysprosium hydroxide precipitated by aqueous ammonia from the nitrate solution at $\mathrm{pH} 7$. 


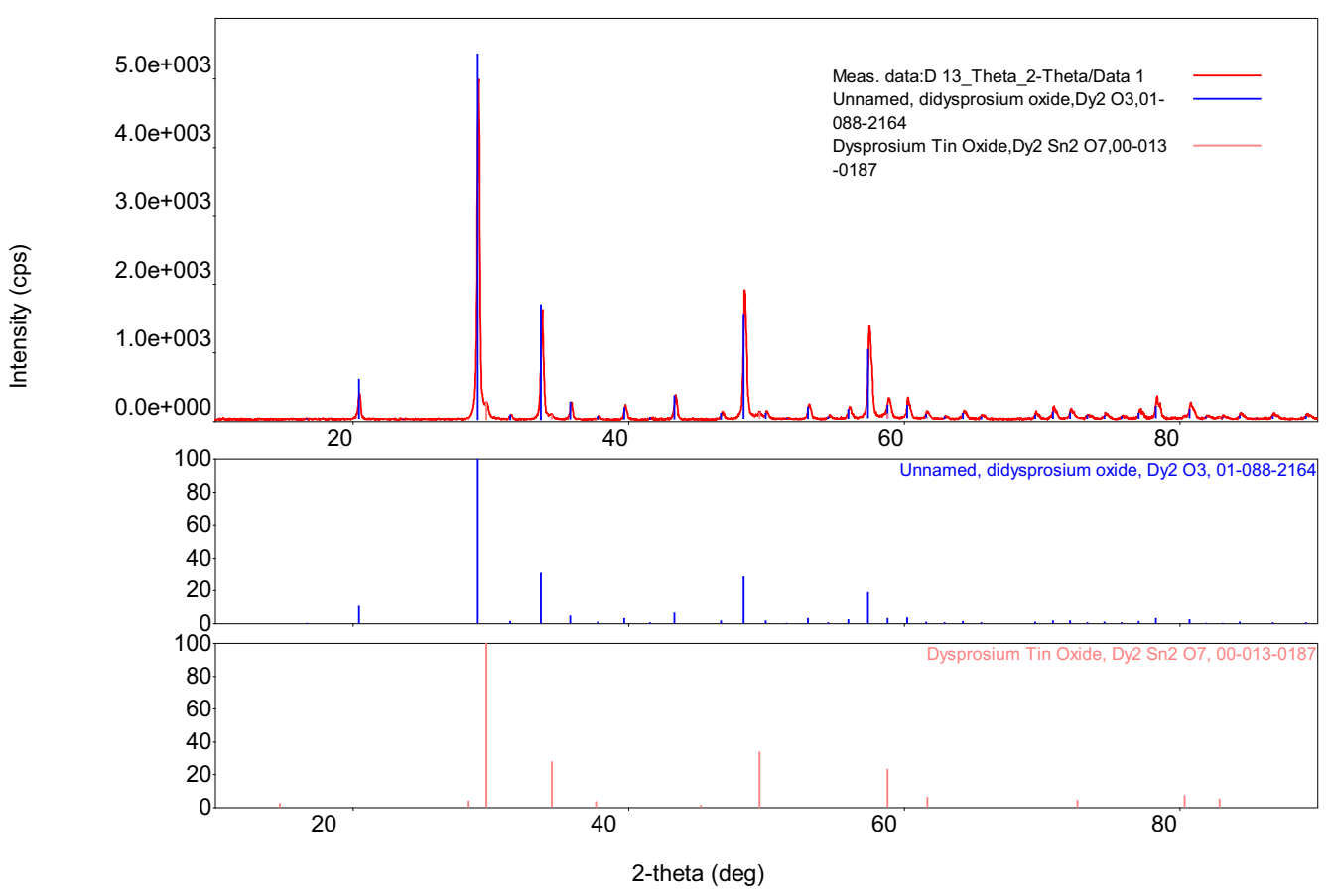

Fig. 3. X-Ray diffraction pattern of the powder obtained by heat treatment at $1000{ }^{\circ} \mathrm{C}$ for 1 hour of dysprosium and tin hydroxides coprecipitated by aqueous ammonia from the nitrate solutions at $\mathrm{pH} 7$.

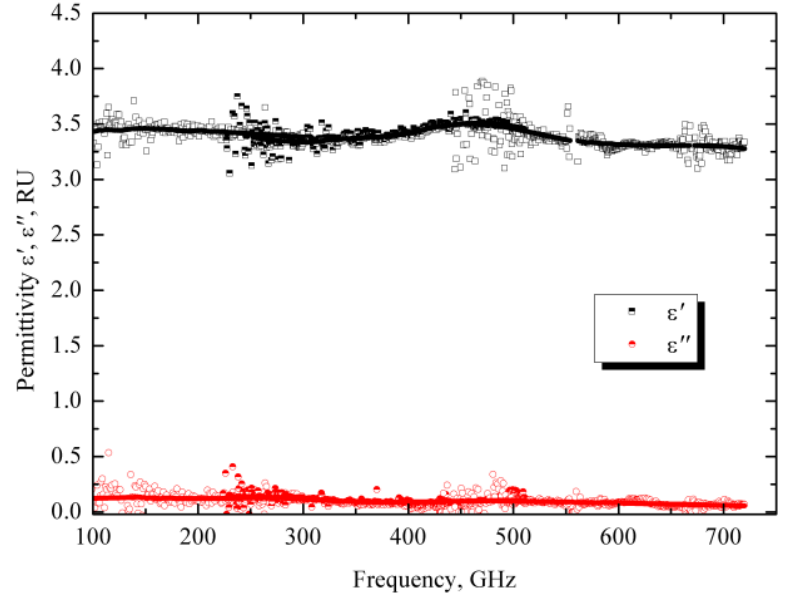

Fig. 4. Complex permittivity spectra of the dysprosium oxide samples.

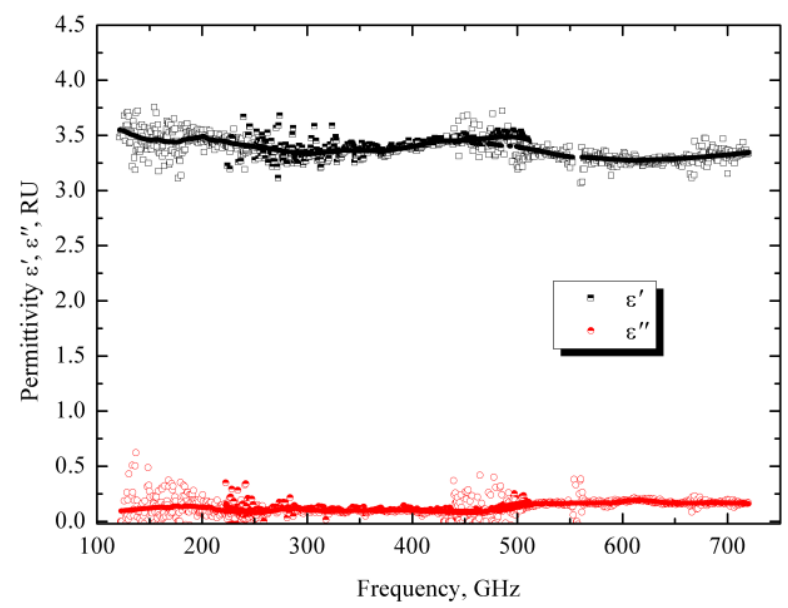

Fig. 5. Complex permittivity spectra of tin-doped dysprosium oxide samples.
This behavior of $\mathrm{CP}$ provides evidence that the studied materials exhibit electronic polarization, which is typical for dielectrics. No differences between the samples of pure dysprosium oxide and those doped with tin are detected, as opposed to indium oxide doped with tin, for which a sharp decrease in the real part of $\mathrm{CP}$ and high values of the imaginary part of $\mathrm{CP}$ are observed at low frequencies [10] indicating the presence of a conductive fraction - free electrons. High values of the $\mathrm{CP}$ components are also retained at high frequencies (Figure 6).

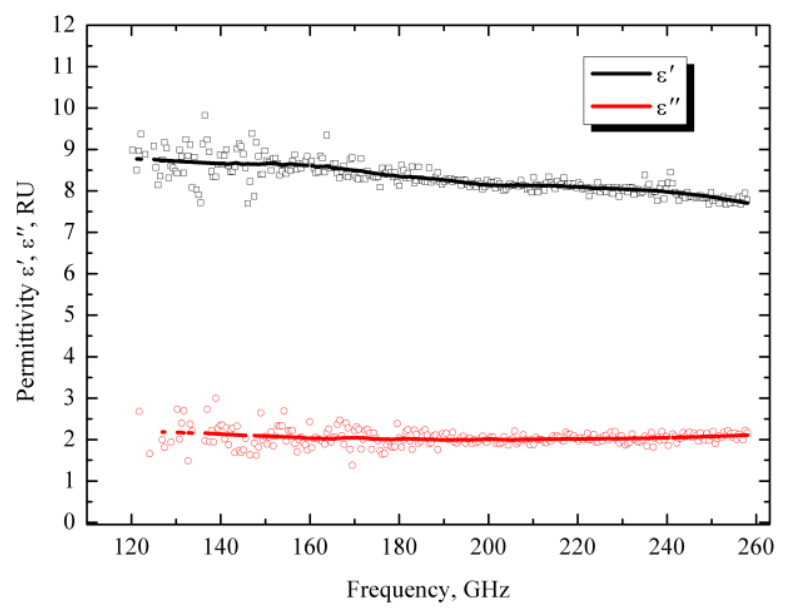

Fig. 6. Complex permittivity spectra of tin-doped indium oxide samples.

These differences affect the components of the electromagnetic response: transmittance $T$, reflectance $R$, and absorption factor $A$ related by the formula $1=R+T+A$. These components define the scope of practical applications of the materials under the study, 
such as screening devices to reduce the level of electromagnetic radiation that is necessary to solve the problems of electromagnetic compatibility, reduction of radio visibility, protection of biological objects, and creating anechoic chambers.

Experimental study of the electromagnetic response components (Figures 7 and 8) shows that from 65 to $80 \%$ of the incoming electromagnetic energy is propagated through the samples of pure and tin-doped dysprosium oxide with a very weak absorption, i.e. the screening properties are determined by the reflectance of samples. On the other hand, the doped indium oxide has a greater absorption of electromagnetic energy that is converted into the heat by the movement of free electrons caused by the electromagnetic field. The transmission factor decreases from 0.3 to 0.15 in the selected frequency range at relatively low reflectance values (Figure 9).

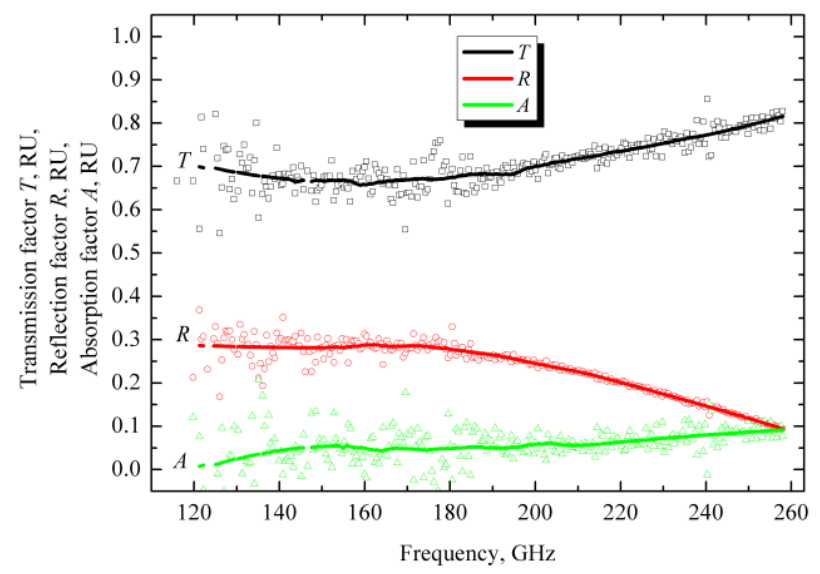

Fig. 7. Spectra of the electromagnetic response components of dysprosium oxide samples: $T$ - transmission factor; $R$ reflection factor; $A$-absorption factor.

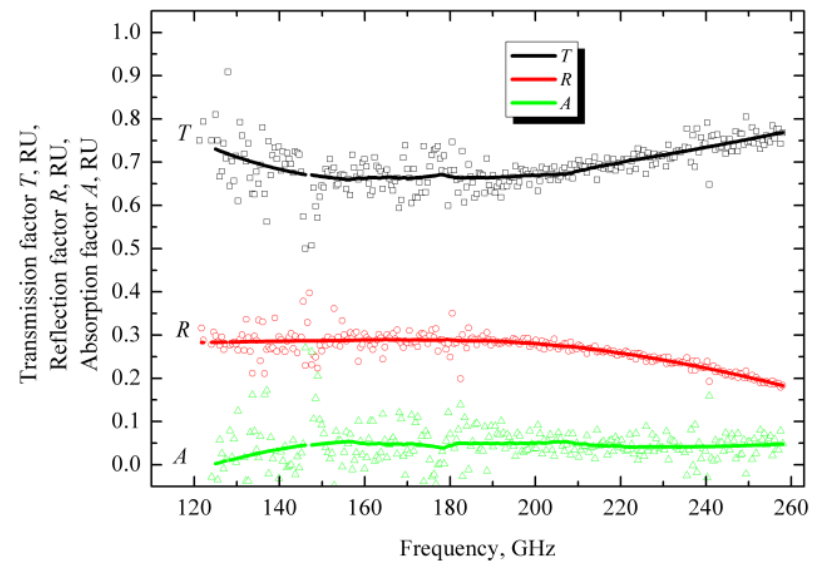

Fig. 8. Spectra of the electromagnetic response components of tin-doped dysprosium oxide samples: $T$-transmission factor; $R$ - reflection factor; $A$ - absorption factor.

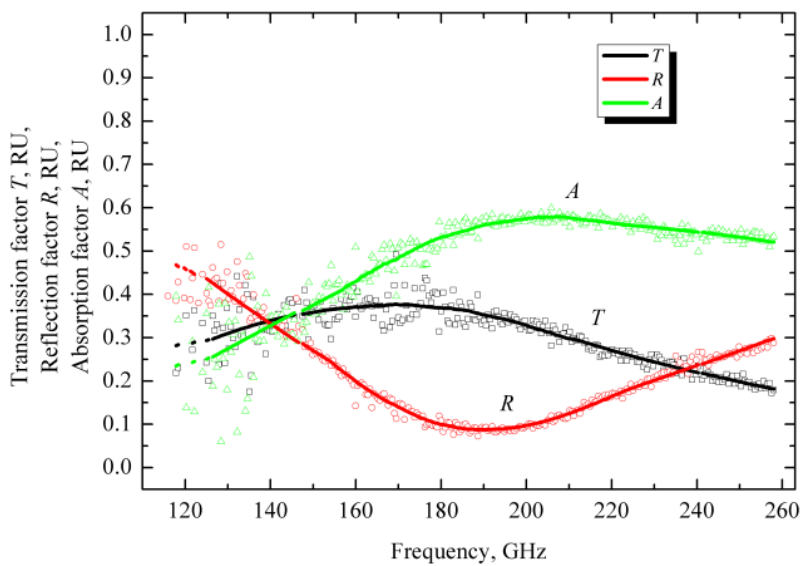

Fig. 9. Spectra of the electromagnetic response components of tin-doped indium oxide samples: $T$ - transmission factor; $R-$ reflection factor; $A$ - absorption factor.

The absence of free electrons in dysprosium oxide doped with tin is confirmed by the absence of the plasma resonance in the spectral curves of the diffuse reflection factor of the tin-doped dysprosium oxide powder. This resonance is, however, observed in tin-doped indium oxide (Figure 10).

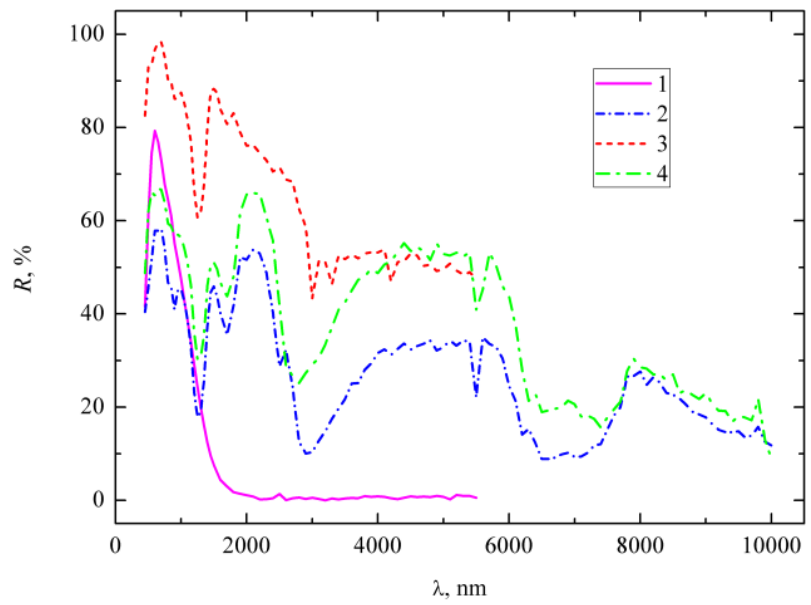

Fig. 10. Spectra of the diffuse reflection of powders of tindoped indium oxide (1), tin-doped dysprosium oxide (2), pure indium oxide (3), and pure dysprosium oxide (4).

\section{Summary and conclusion}

Thus, doping of dysprosium oxide with tin has no effect on the electromagnetic and optical characteristics of the material. At the same time, doping of indium oxide with the same amount of tin results in a significant increase of the CP components and an appearance of the plasma resonance in its diffuse reflection spectra. The data obtained indicate the appearance of charge carriers with the concentration of $10^{21} \mathrm{~cm}^{-3}$ in the materials based on the dispersed ITO with the tin content of $10 \mathrm{wt} . \%$.

The studies showed that the composite material based on the dispersed ITO doped with tin effectively interacts with the EHF electromagnetic radiation and can be used in the screening devices reducing the level of electromagnetic radiation, which is necessary for solving the problems of electromagnetic compatibility, reduction 
of radio visibility, protection of biological objects, and creating the anechoic chambers.

\section{Acknowledgments}

The work was supported by the Tomsk State University Competitiveness Improvement Programme, registration No. 8.1.23.2015.

\section{References}

1. H. S. Nalwa, Handbook of Nanostructured Materials and Nanotechnology (Academia Press, San Diego, 1999).

2. M. Mikuśkiewicz, M. Stopyra, G. Moskal, Arch. Metall. Mater., 61, 2B, 965-969 (2016).

3. R. C. Layland, S. L. Kirkland, H. C. Loye, J. Solid State Chem., 139, 1 p. 79-84 (1998).

4. V. D. Risovany, A. V. Zakharov, E. M. Muraleva, et al., J. Nucl. Mater. 355, 1, 163-170 (2006).

5. Q. Xu, W. Pan, J. Wang, et al., J. Amer. Ceram. Soc. 89, 1, 340-342 (2006).

6. D. S. Ginley, Handbook of transparent conductors (Springer, New York, 2010).

7. V. I. Suslyaev, G. E. Dunaevskyi, E. V. Emelyanov, G. E. Kuleshov, Proceedings of the higher educational institutions. Physics (in Russian Izvestiya vuzov. Physica), 54, 9/2, 138-146 (2011).

8. T. D. Malinovskaya, V. I. Suslyaev, S. V. Melentyev, K. V. Dorozkin, Russ. Phys. J. 57, 8, 1094-1098 (2014).

9. Y. P. Egorov, T. D. Malinovskaya, E. P. Naiden, V. I. Sachkov, E. I. Sachkova, Chem. Sustain. Dev. 10, 679-685 (2002).

10. T. D. Malinovskaya, V. I. Suslyaev, V. A. Zhuravlev, S. V. Melentyev, E. Yu. Korovin, K. V. Dorozhkin, V. V. Zhek, S. V. Pavlov, Proceedings of the higher educational institutions. Physics (in Russian Izvestiya vuzov. Physica), 59, 9, 168-169 (2016). 